Z. klin. Chem. u. klin. Biochem.

7. Jg., S. 301-305, Mai 1969

\title{
Zur Reaktivierung der Acetylcholinesterase und Cholinesterase
}

\author{
Titrimetrische Untersuchungen an Hirnbomogenat und Plasma nach Tabun-, Sarin- und Somanbemmung in vitro
}

Von P. BACK

Aus der Akademie des Sanitäts- und Gesundbeitsvesens der Bundeswebr (Kommandeur: Generalarzt Dr. C. Merkle)

(Eingegangen am 27. Januar 1969)

Untersuchungen der Reaktivierbarkeit Tabun-, Sarin- und Somangehemmter Acetylcholinesterase und Plasmacholinesterase durch Pyridiniumaldoxime erfordern eine Meßmethode, die unabhängig ist von Extinktionsänderungen und Ånderungen des $\mathrm{pH}$-Wertes des Reaktionsmediums, welche durch den Reaktivatorzusatz hervorgerufen werden. Die automatische titrimetrische Messung nach der $\mathrm{pH}-$ Stat-Methode exlaubt die exakte Bestimmung von Enzymaktivitäten auch nach Reaktivatorzusatz und bietet den Vorteil, schnell und wenig aufwendig zu sein. Reaktivierungen konnten nach Tabun-, Sarin- und Somanhemmung teilweise erzielt werden, unterliegen jedoch so vielen einschränkenden Bedingungen, daß der Reaktivierungsversuch als spezifischer diagnostischer Test für eine vorliegende Alkylphosphatvergiftung im klinischen und forensischen Bereich von fraglichem Wert erscheint.

\section{The reactivation of acetylcholinesterase and cholinesterase. A titrimetric investigation of brain bomogenate and plasma after in vitro inbibition by Tabun, Sarin and Soman}

Studies on the reactivation of tabun, sarin- and somaninhibited acetylcholineesterase and cholineesterase by pyridiniumaldoximes should be performed by a method, which is independent of colorimetric changes and changes of the $\mathrm{pH}$ in the reaction mixture introduced by the reactivator. The titrimetric pH-stat-method seems to be the only reliable method to avoid errors in the measurement of true enzyme-activity after addition of pyridiniumaldoximes. Reactivation could be shown for acetylcholinesterase in brain homogenates. of rats after preincubation with tabun, sarin and soman and for cholinesterase in human plasma after sarin inhibition in vitro. The reactivation however is subject to several conditions, which do not allow a general application of this reaction as a diagnostic tool in the clinical and forensic laboratory for the detection of alkylphosphate poisoning.

Als spezifisches Nachweisverfahren bei Verdacht auf Alkylphosphatvergiftung wurde die Bestimmung der Cholinesteraseaktivität im Blutplasma sowie der Acetylcholinesteraseaktivität vor und nach Zusatz von Oximreaktivatoren vorgeschlagen (1). Danach soll die Diagnose einer Alkylphosphatvergiftung gesichert sein, wenn eine Erhöhung der Enzymaktivität nach Reaktivatorzusatz gefunden werden kann.

$\mathrm{Da}$ wir in keinem Falle in Blutproben ${ }^{1}$ ) von Patienten mit anamnestisch gesicherter Alkylphosphatvergiftung eine Aktivitätserhöhung der Plasmacholinesterase ${ }^{2}$ ) und der Erythrocytenacetylcholinesterase ${ }^{2}$ ) nach Zusatz von Toxogonin (Oxy-bis-(4-hydroximinomethyl-1-pyridinium)-dichlorid, Obidoxim) finden konnten, erschien es wünschenswert, nach kritischer Prüfung verschiedener Bestimmungsmethoden für Acetylcholinesterase die Frage der Reaktivierungsmöglichkeiten durch kinetische Untersuchungen an Tabun-, Sarin- und Somangehemmten Enzymen zu klären. Tabun, Sarin und Soman gelten als die wirksamsten Acetylcholinesteraseinhibitoren aus der Gruppe der Alkylphosphate.

\section{Methodik}

Als Enzymquelle für Plasma-ChE diente Blutplasma vom Menschen, welches nach Zentrifugieren von frisch entnommenem, heparinisierten Blut gewonnen wurde.

1) Die Blutproben wurden uns durch Hcrrn Dr. M. v. CuAraranN (Klinikum rechts der Isar der TH München) zur Verfügung gestellt, wofür wir auch an dieser Stelle unseren Dank aussprechen möchten.

2) Plasmacholincstcrase (EC 3.1.1.8) = Plasma-ChE; Erythrocytcnacetyleholinestcrase (EC 3.1.1.7) $=$ AChE.
Hirnbomogenat, als Enzymquelle für $\mathrm{AChE}$ (Acetylcholinacetylhydrolase, EC 3.1.1.7), wurde hergestellt durch Homogenisieren von $16 \mathrm{~g}$ frisch entnommenem, eisgekühlten Rattengehirn mit $24 \mathrm{ml} 0,9$ proz. NaCl-Lösung im Potter-Elvehjem-Homogenisator. Nach Filtrieren durch drei Schichten Verbandgaze wurde das Homogenat in mehreren Portionen eingefroren und bei $-17^{\circ}$ aufbewahrt. In tiefgefrorenem Zustand war das Enzym über drei Monate ohne Aktivitätsverlust haltbar.

Für Inkubationsserien wurde jeweils eine Portion aufgetaut und nach längstens 6 Stdn. Aufbewahrung im Eisbad verwendet. Eine Aktivitätsabnahme war bei diesem Vorgehen nicht feststellbar.

Die Aktivitätsbestimmungen wurden nach der $\mathrm{pH}-\mathrm{Stat}-\mathrm{Methode}$ $(2,3)$ mit Hilfe der Apparatur von Radiometer, Kopenhagen in Miktogefäßen bei $37^{\circ}$ unter ständigem Rühren des Reaktionsansatzes durchgeführt. Die Titration erfolgte unter Luftabschluß mit der Autobürette ABU 1, die Registrierung durch den Titrigraph.

Der Reaktionsansatz enthielt für die Bestimmung der Plasma-CbE in $4,2 \mathrm{~m} l$ Endvolumen $2,0 \mathrm{~m} /$ Pufferlösung $(0,006 \mathrm{M} \mathrm{Na}$-diäthylbarbiturat, $0,001 \mathrm{M} \mathrm{KH}_{2} \mathrm{PO}_{4}, 0,3 \mathrm{M} \mathrm{NaCl}$ ), eingestellt auf $\mathrm{pH} 7,8$ mit $0,1 \mathrm{~N} \mathrm{HCl}, 1,6 \mathrm{~m} /$ dest. Wasser, $0,4 \mathrm{~m} /$ Plasma und $0,2 \mathrm{~m} l$ einer $0,06 \mathrm{M}$ Acetylcholinchloridlösung in dest. Wasser.

Die Zusammensetzung des Reaktionsansatzes für die Bestimmung der Hirm-ACbE bestand aus $2,0 \mathrm{ml}$ Pufferlösung $(0,005 \mathrm{Mr} \mathrm{Na}$ diäthylbarbiturat, $0,001 \mathrm{M} \mathrm{KH}_{2} \mathrm{PO}_{4}, 0,15 \mathrm{M} \mathrm{KCl}$ ), eingestellt auf pH 7,4 mit $0,1 \mathrm{~N} \mathrm{HCl}, 1,6 \mathrm{ml}$ dest. Wasser, $0,4 \mathrm{~m} /$ Hirnhomogenat und $0,2 \mathrm{~m} /$ einer $0,05 \mathrm{M}$ Acctylcholinchloridlösung in dest. Wasser. Die Substratlösungen wurden täglich frisch hergestellt und im Eisbad gehalten.

Die enzymatisch freigesetzte Essigsäure wurde mit ciner $0,005 \mathrm{~N}$ $\mathrm{NaOH}$, welche in kurzen Abständen erneucrt wurde, titriert.

Die Reaktionen wurden nach 5 Min. Aquilibricrungszeit durch Zusatz von Substrat gestartet. Linearer Vcrlauf der Enzymreaktion war während der crsten Minuten gegeben.

In den Hemmversuchen mit verschicdenen Konzentrationen Tabun (Dimethylaminocyanphosphorsäureäthylester), Sarin (Methylfuorphosphonsäurcisopropylester) und Soman (Methylfluor- 

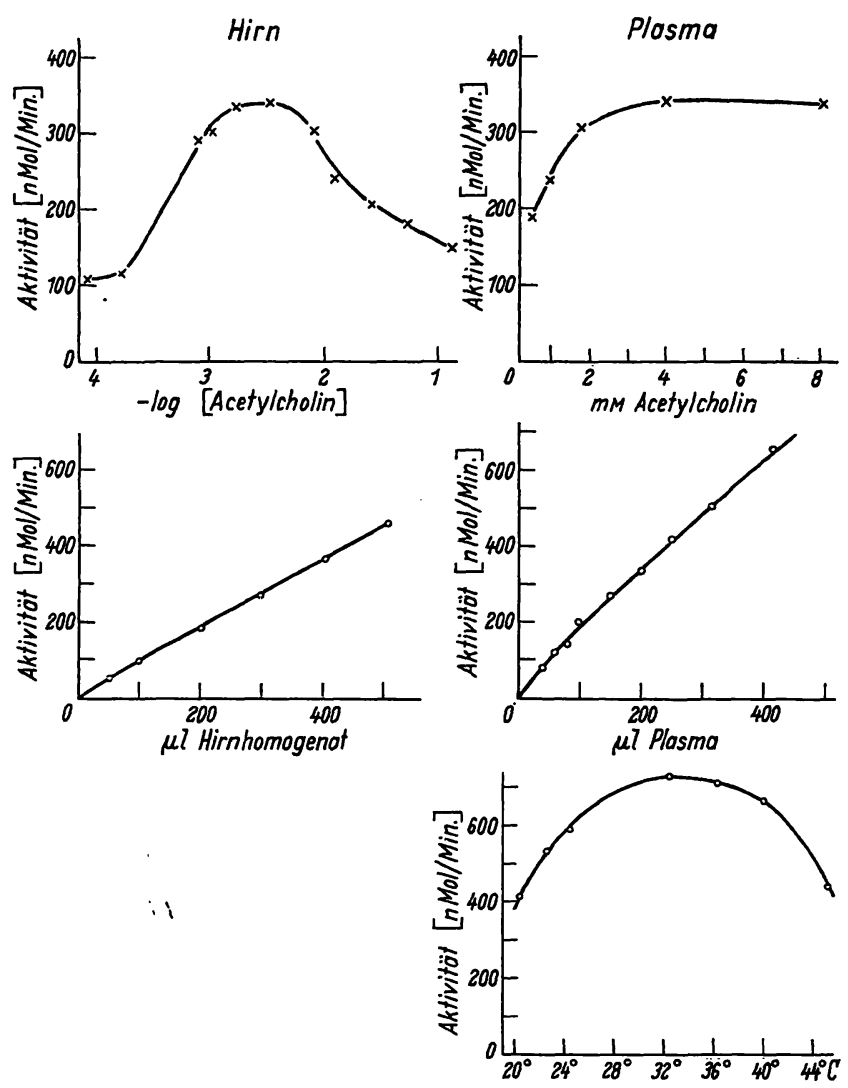

Abb. 1

Substrat-, Enzym- und Temperaturabhängigkeiten der AChE in Hirnhomogenat und der Plasma-ChE
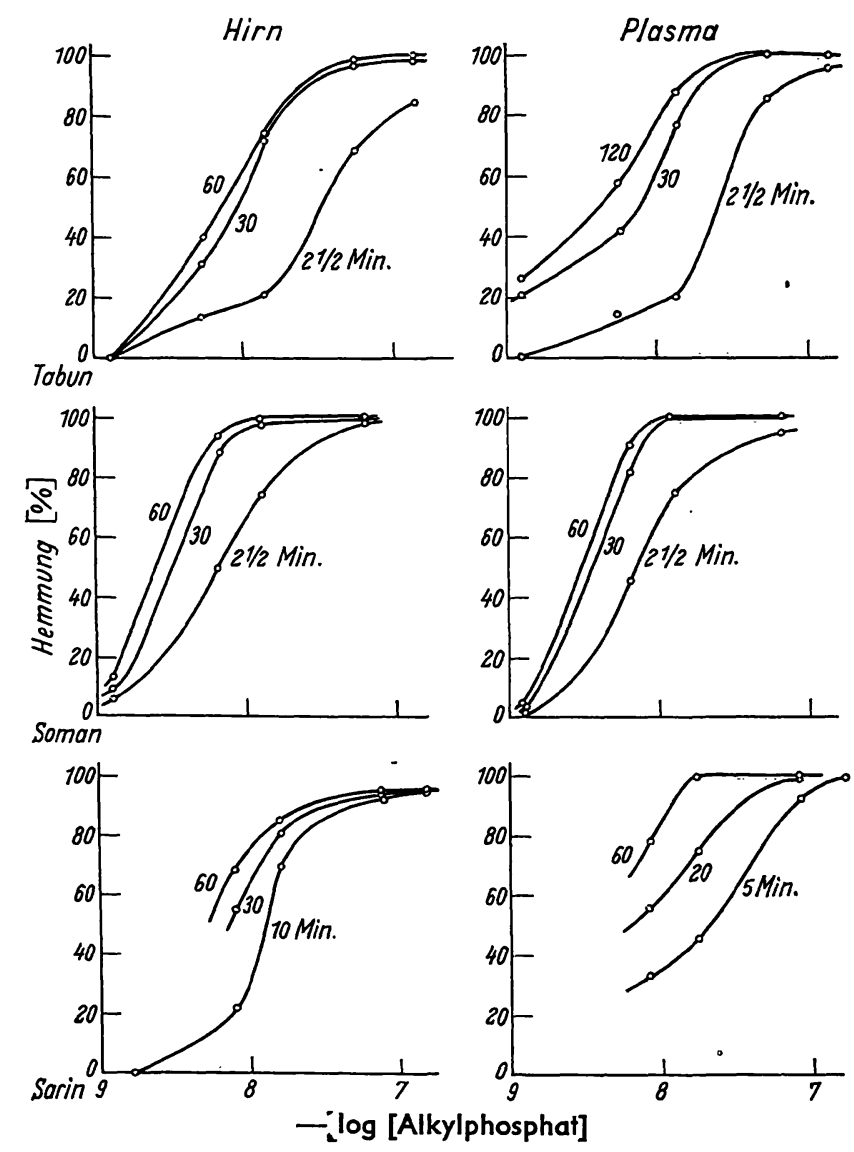

Abb. 2

Hemmung von $A C h E$ und Plasma-ChE durch Tabun, Soman und Sarin in Abhängigkeit von der Alkylphosphatkonzentration und von der Vorinkubationszeit mit den Alkylphosphaten phosphonsäurepinakolylester) wurde der Reaktionsansatz ohne Substrat verschieden lange Zeit bei $37^{\circ} \mathrm{mit}$ dem jeweiligen Alkylphosphat vorinkubiert. Kontrollansätze enthielten $0,1 \mathrm{~m} l$ Isopropanol, das Lösungsmittel für die Alkylphosphate. Die Aktivität der gehemmten Enzyme wurde nach Ablauf der Votinkubationszeit durch Substratzusatz getestet. In Reaktivierungsversuchen effolgte drei Min. nach Substratzugabe der Zusatz von $0,1 \mathrm{ml}$ des jeweiligen Oximreaktivators 2-PAM (2-Hydroxyiminomethyl-1-methylpyridinium-jodid), P-2-S (2-Hydroxyiminomethyl-1-methylpyridiniummethylsulfonat) und Obidoxim (Oxybis-(4-hydroxyiminomethyl-1-methylpyridinium)-dichlorid, Toxogonin). Die Reaktion wurde so lange registriert, bis linearer Verlauf eintrat.

Die Auswertung erfolgte graphisch. Die Berechnung der Hemmungs- und Reaktivierungsaktivitäten wurde jeweils auf die Aktivität des Kontrollansatzes bezogen und in Prozent der Kontrollaktivität ausgedrückt.

\section{Ergebnisse}

Die im klinischen Laboratorium häufig angewandten kolorimetrischen Bestimmungsmethoden der Plasma$\mathrm{ChE}$ und der AChE mit Acetyl-thiocholin als Substrat (4), sowie die o-Nitrophenylbutyrat verwendende Methode $(5,6)$ erweisen sich als ungeeignet für Untersuchungen mit Reaktivatorzusatz, weil Pyridiniumaldoxime aus der Enolform schon in schwach alkalischem Medium unter Protonabspaltung in eine Zwitterionenform übergehen. Diese zeigt Mesomerie mit einer stark farbgebenden Form, wie für 2-PAM (7) und in eigenen Versuchen auch für Obidoxim nachgewiesen wurde. Es resultiert eine $\mathrm{pH}$ - und konzentrationsabhängige Gelbfärbung, welche Messungen im Bereich um $405 \mathrm{~nm}$ stört.

Die manometrische Messung (8) beansprucht mehrere Kontrollansätze, längere Vorinkubationszeiten und Reaktionszeiten, wodurch Messungen der Änderungen von Maximalgeschwindigkeiten erschwert werden, welche für AChE nur in dem Substratkonzentrationsbereich von $2-2,5 \mathrm{~mm}$ Acetylcholin gemäß 0. Ordnung verlaufen. Bei höheren Substratkonzentrationen tritt Substrathemmung ein (9). Wie schon erwähnt, werden Protonen aus dem Reaktivator in schwach alkalischem Medium frei, was bei den Messungen berücksichtigt werden muß.

Diskontinuierliche Bestimmungsmethoden wurden nicht geprüft, weil kinetische Messungen mit ihnen nur sehr bedingt durchzuführen sind.

Für die hier mitgeteilten Untersuchungen wurde deshalb die pH-Stat-Methode $(2,3)$ gewählt, welche durch automatische Titration eine kontinuierliche Enzymaktivitätsmessung innerhalb der ersten Minuten nach Substratzusatz gewährleistet. Abbildung 1 zeigt Substrat- und Enzymabhängigkeiten für $\mathrm{AChE}$ aus Rattengehirn und Plasma-ChE unter den angewandten Versuchsbedingungen.

\section{Hemmversuche}

Tabun, Sarin und Soman wurden den Enzymansätzen in isopropanolischer Lösung in Konzentrationen von 1 bis $100 \mathrm{nM}$, im Falle von Sarin auch bis zu einer Konzentration von $10 \mu \mathrm{M}$, bezogen auf den Gesamtreaktionsansatz, zugegeben. Bei $37^{\circ}$ wurden die En- 
zympräparationen verschieden lange Zeit mit dem Alkylphosphat vorinkubiert. Die resultierenden Hemmungskurven sind aus Abbildung $2 \mathrm{zu}$ ersehen. Sie zeigen Abhängigkeit des $\mathrm{pI}_{50}$, des negativen Logarithmus jener Hemmstoffkonzentration, welche zu 50proz. Hemmung der Enzymaktivität führt, von der Dauer der Vorinkubation mit dem Alkylphosphat. Nach $2^{1} / 2$ Min. Vorinkubationszeit beträgt der $\mathrm{pI}_{50^{-}}$ Wert für Soman 8,2, der $\mathrm{pI}_{50}$-Wert für Tabun 7,5.

\section{Reaktivierungsversuche}

Der Reaktivatorzusatz erfolgte zum Enzym-InhibitorSubstrat-Gemisch, nachdem die Reaktion drei Min. abgelaufen war. Die drei Oximreaktivatoren 2-PAM, P-2-S und Obidoxim, deren Strukturformeln in der Enolform Abbildung 3 wiedergibt, wurden getestet.<smiles>C[n+]1ccccc1/C=N/O</smiles>

2-PAM<smiles>C[n+]1ccccc1/C=N/O</smiles><smiles>O/N=C/c1cc[n+](COC[n+]2ccc(Cl)cc2)cc1</smiles>

Abb. 3

Strukturformeln der verwendeten Reaktivatoren

Es stellte sich heraus, daß Obidoxim, sofern überhaupt Reaktivierungseffekte $z u$ beobachten waren, in allen Fällen die größte Wirkung zeigte (Abb. 4). Daraufhin beschränkten sich alle weiteren Untersuchungen auf dieses Oxim allein. Die Oximkonzentration im Ansatz betrug 0,1 mM. Ein geringer Hemmeffekt durch Obidoxim am nicht mit Alkylphosphat vorinkubierten Enzym wurde beobachtet. Er lag bei $5-10 \%$ der Kontrollaktivität für Plasma-ChE und AChE.

Die Untersuchungen am Hirngewebe der Ratte zeigten einen Reaktivierungseffekt durch Obidoxim bei Tabunkonzentrationen, welche über $72 \mathrm{~nm}$ liegen und zu einer Hemmung von mehr als $80 \%$ der Enzymaktivität geführt hatten (Abb. 5).

Die Tabunhemmung von Plasma-ChE ließ sich in keinem Fall durch Obidoxim auch nur teilweise aufheben. Obidoximzusatz führte lediglich zu zusätzlicher Aktivitätssenkung.

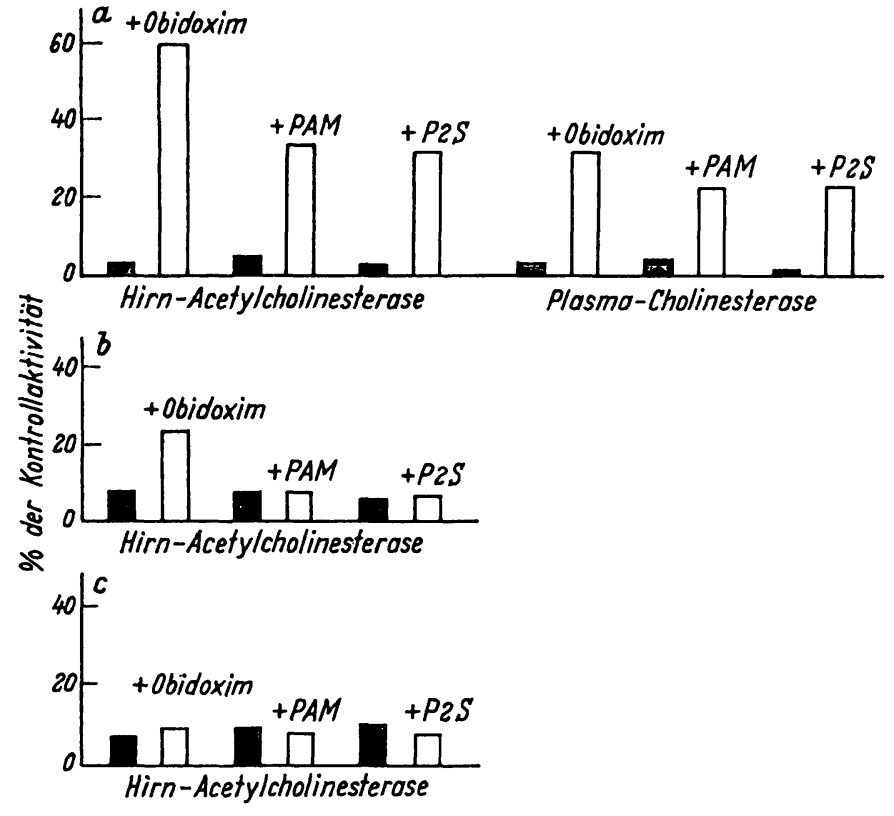

Abb. 4

Reaktivierungseffekte von $0,1 \mathrm{mM}$ Obidoxim, 2-PAM und P-2-S unter gleichen Bedingungen der Vorinkubation mit Alkylphosphat a) Sarin, $30 \mathrm{Min} ., 83 \mathrm{nM}$; b) Tabun, 150 Sek., $72 \mathrm{mM}$; c) Soman, $30 \mathrm{Min}$.,

Nach Sarinhemmung kann Obidoximzusatz eine Reaktivierung der Plasma-ChE bewirken (Abb. 6), solange die Sarinkonzentration unter $10 \mu \mathrm{M}$ liegt. Die Reaktivierung ist nicht abhängig von der Dauer der Vorinkubation mit dem Alkylphosphat, sondern allein von der Sarinkonzentration. Bei Extrapolation einer durch die Reaktivierungsmittelwerte verlaufenden $\mathrm{Ge}$ raden nach Auftragung gegen den Logarithmus der Sarinkonzentration ergibt sich ein Schnittpunkt mit der Abszisse (log Sarinkonzentration) bei dem Wert - 5 . Bei einer Sarinkonzentration von $10^{-5} \mathrm{M}$ sollte demnach keine Reaktivierung mehr möglich sein. In der Tat gaben Versuche mit einer Sarinkonzentration von $16,6 \mu \mathrm{M}$ kaum noch einen Reaktivierungseffekt nach Obidoximzugabe.

Im Hirnhomogenat liegen die Verhältnisse ähnlich. Jedoch ist eine Abnahme des Reaktivierungsgrades mit zunehmender Dauer der Vorinkubation mit Sarin zu erkennen. Nach Vorinkubation mit $16,6 \mu \mathrm{M}$ Sarin ist nur noch eine geringe Reaktivierung durch Obidoxim in der Konzentration von $0,1 \mathrm{~mm}$ zu erzielen. Auch höhere Obidoximkonzentrationen ergeben keine Steigerung des erreichten Reaktivierungsgrades.

Abbildung 7 zeigt die Ergebnisse aus den Versuchen mit Soman. In einem engen Konzentrationsbereich von 30-60 nм Soman ist eine geringe Reaktivierung der AChE im Himhomogenat nachzuweisen. Sie erreicht maximal jedoch nur $6 \%$ der Kontrollaktivität. Zunehmende Somankonzentrationen führen wiederum zu einer Abnahme der Reaktivierbarkeit.

In keinem Fall konnte im Plasma nach Somanhemmung der Plasma-ChE eine Reaktivierung erzielt werden, auch nicht bei sehr geringen Somankonzentrationen und nur kurzen Vorinkubationszeiten. Obidoxim wirkte hier lediglich als zusätzlicher Hemmer. 
304

Back:Zur Reaktivierung der Acetylcholinesterase und Cholinesterase

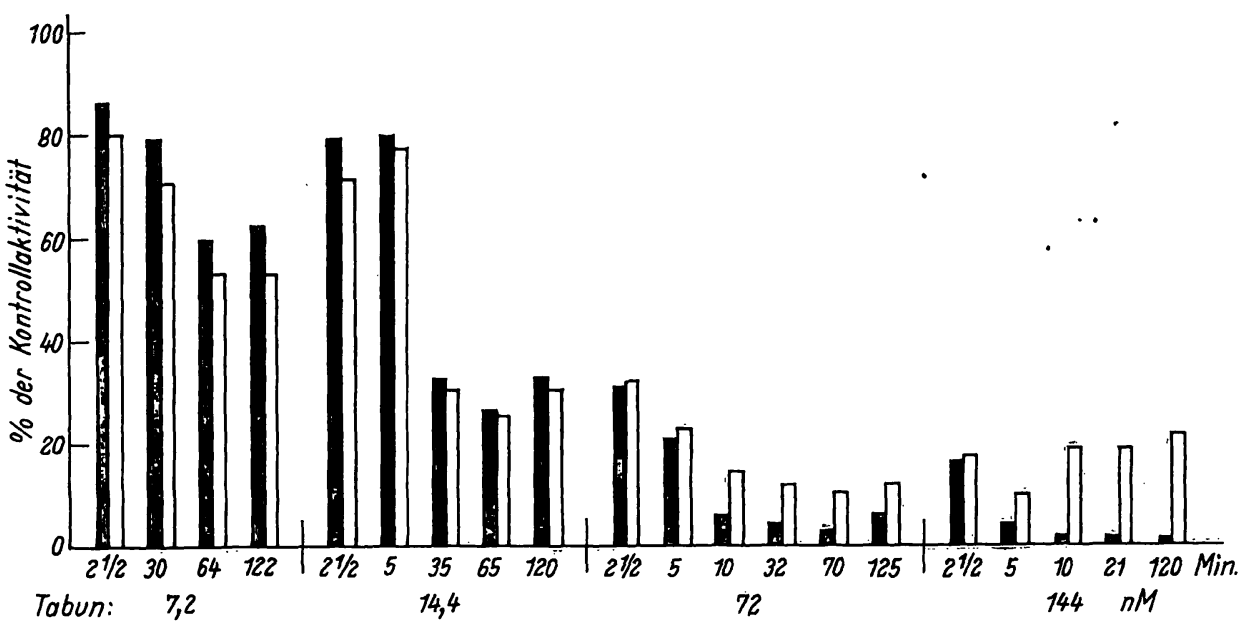

Abb. 5

Hemmung der AChE in Hirnhomogenat der Ratte nach verschiedenen Vorinkubationszeiten mit verschiedenen Konzentrationen von Tabun (schwarze Säulen) und Reakțivierung mit $0,1 \mathrm{~mm}$ Obidoxim (weiße Säulen)

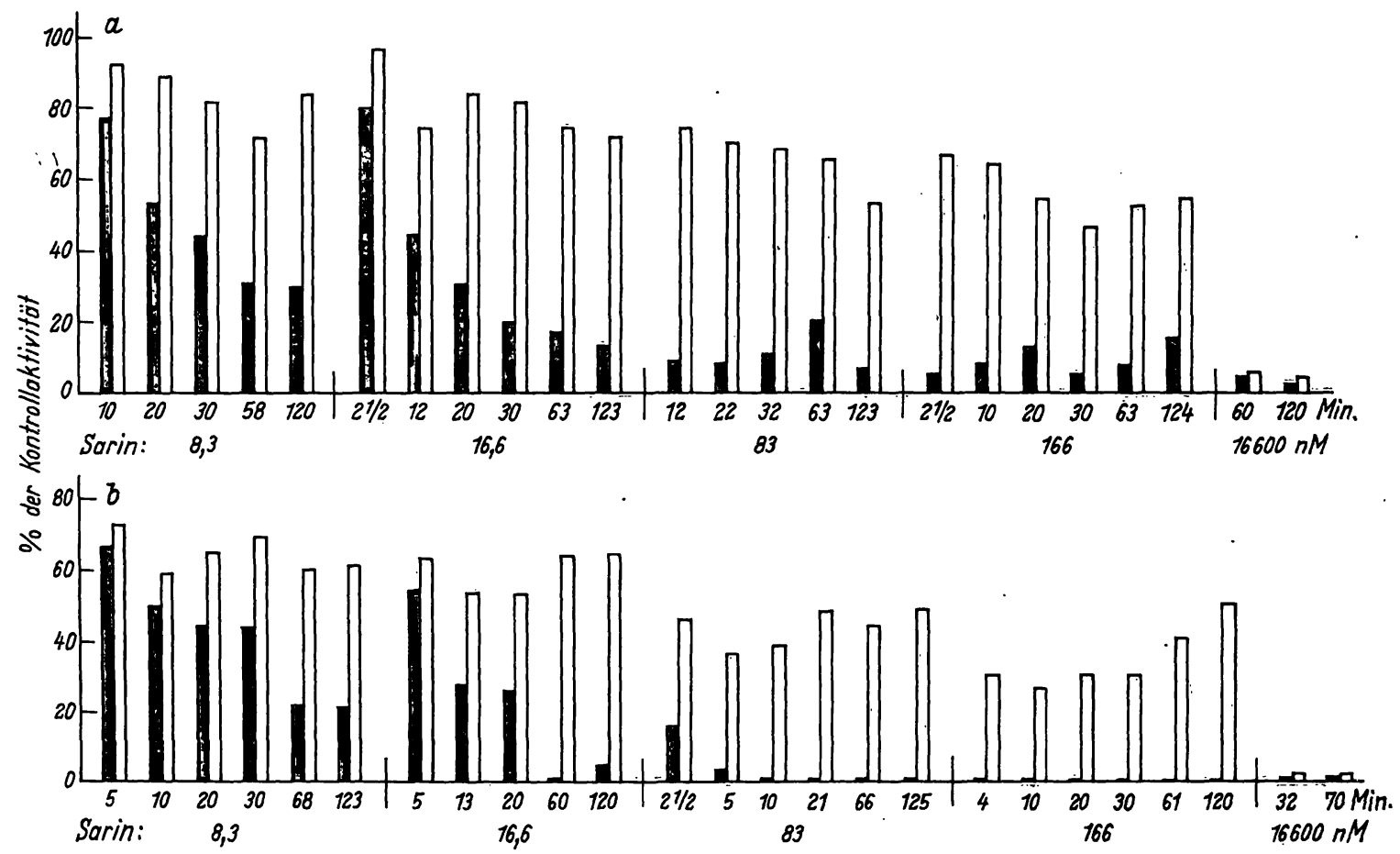

Abb. 6

Hemmung der AChE in Hirnhomogenat (a) und der Plasmacholinesterase des Menschen (b) nach verschieden langen Vorinkubationszeiten mit verschiedenen Konzentrationen von Sarin (schwarze Säulen) und Reaktivierung mit 0,1 mM Obidoxim (weiße Säulen)

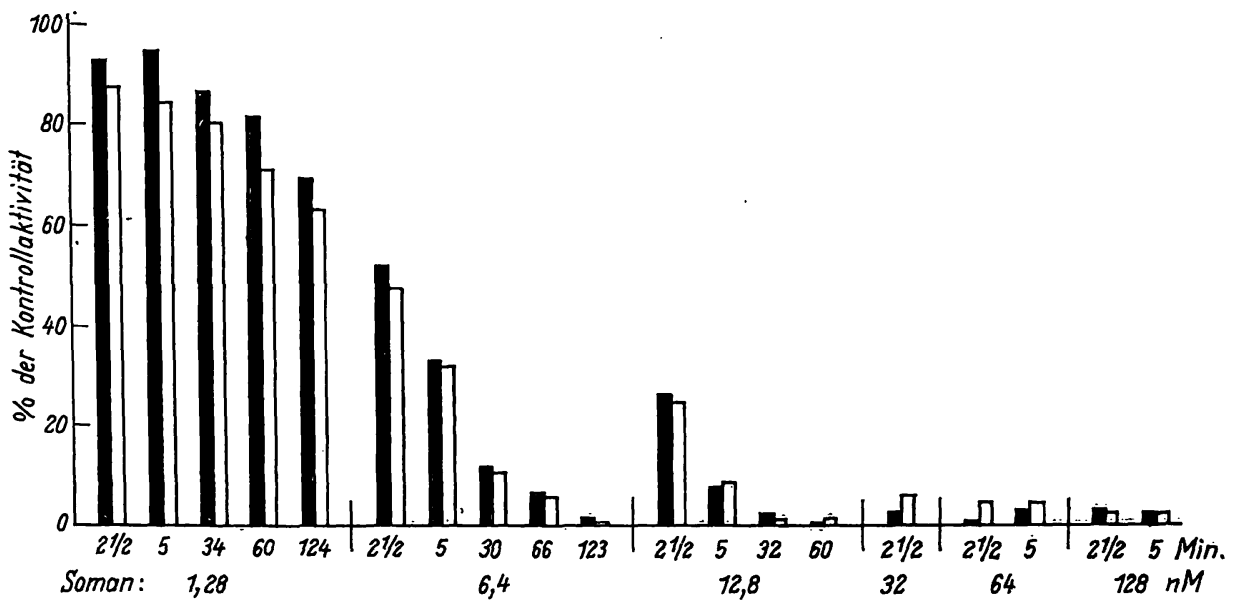

Abb. 7

Hemmung der AChE in Hirnhomogenat der Ratte nach verschieden langen Vorinkubationszeiten mit verschiedenen Konzentrationen von Soman (schwarze Säulen) und Reaktivierung mit 0,1 mM Obidoxim (weiße Säulen)

Z. klin. Chem. u. klin. Biochem. / 7. Jahrg. 1969 / Heft 3 


\section{Diskussion}

Wie für 2-PAM nachgewiesen wurde, kann ein Reaktivator vom Typ der Pyridiniumaldoxime mit seinem kationischen Zentrum durch Konkurrenz um die anionische Bindungsstelle der AChE (10) als reversibler Inhibitor des Enzyms wirken (11). Diese Eigenschaft kann im Falle von Obidoxim, einem Molekül mit zwei solchen kationischen Zentren, zur Erklärung der ihm eigenen Hemmwirkung herangezogen werden. Diese Hemmwirkung konnte auch bei Tabun- und Somaneinwirkung gefunden werden, solange nicht-phosphorylierte Enzymmoleküle in genügender Zahl vorhanden waren, d. h. solange die Enzymaktivität noch mehr als $20 \%$ bzw. $5 \%$ der Kontrollaktivität betrug. Lag die Enzymaktivität bei dem letztgenannten $\mathrm{Al}$ kylphosphat unter $5 \%$, so ließ sich eine geringe Reaktivierung nachweisen, wenn die Vorinkubationszeit so kurz gehalten wurde, daß der "Alterungsprozeß", durch den das phosphorylierte Enzym in einen nicht mehr reaktivierbaren Zustand übergeht (12-15), noch nicht weit fortgeschritten sein konnte (16). Der Alterungsprozeß der Saringehemmten AChE im Hirngewebe läßt sich aus dem Abfall der Reaktivierungswerte nach Obidoximzusatz, welcher bei gleicher Alkylphosphatkonzentration mit zunehmender Dauer der Vorinkubationszeit eintrat, ablesen. Nach Tabunhemmung ließ sich eine Reaktivierung deutlich nachweisen, ohne daß ein Alterungsproze $ß$ im Verlaufe längerer Vorinkubationszeiten $\mathrm{zu}$ erkennen war. Bei Tabun, einem Ester der Phosphorsäure mit dem primären Alkohol Äthanol, fehlt die bei Sarin und Soman vorliegende geradkettige bzw. verzweigtkettige sekundäre Alkoxygruppe, welche von entscheidendem Einfluß auf die Alterungsgeschwindigkeit ist (17).

Die Plasma-ChE war in keinem Falle nach Vorinkubation mit Tabun oder Soman zu reaktivieren.

Für die Abnahme der Reaktivierbarkeit der saringehemmten Enzyme in Plasma und Hirnhomogenat bei steigenden Konzentrationen des Alkylphosphats ist wahrscheinlich die Bildung eines inhibitorisch wirksamen phosphorylierten Reaktivators $(18,19)$, wie er für Sarin und TMB 4 (1,1'-Trimethylen-bis-(4-hydroxyiminomethylpyridinium)-dibromid) beschrieben wurde $(20,21)$, verantwortlich. TMB 4 unterscheidet sich von Obidoxim nur durch das Vorliegen einer Methylengruppe an Stelle der Ätherbrücke.

Eine ausschlaggebende Rolle für die Diagnose einer Alkylphosphatvergiftung kann dem im klinischen oder forensischen Labor durchgeführten. Reaktivierungsversuch an Hand der dargelegten Befunde nicht zugesprochen werden. Reaktivierungsversuche in vitro können in der Regel nicht unmittelbar nach Vergiftungsbeginn durchgeführt werden. Es empfiehlt sich daher für die Klinik mehr, ein Vergiftungsbild an Hand von Verlaufskontrollen der Enzymaktivitäten zu verfolgen.

\section{Literatur}

1. FrIedberg, K. D und F. SAKat, Dtsch. Zschr. gerichtl. Med. 47, 580 (1958). - 2. Larsson, L. und B. Hansen, Svensk. kem. Tidskr. 68, 521 (1956). - 3. JACOBSEN, C. F., J. LÉONIS, K. LiNDERSTROM-LANG und M. OtTENSEN, in Methods of biochemical analysis Bd. IV S. 171 Hrsg. D. Glick, Interscience, New YorkLondon (1957). - 4. Eliman, G. L., K. D. Courtney, V. Andres. jt. und R. M. Featherstione, Biochem. Phämacol. 7, 88 (1961). 5. Main, A. R., K. E. Miles und P. E. Braid, Biochem. J. 78, 769 (1961). - 6. GeLDMACHER - v. MaLLINCKRoDT, M. und J. KaISER, diese Z. 6, 141 (1968). - 7. GibBON, S. L. und J. L. WAY, J. Chromatogr. 26, 202 (1967). - 8. Ammon, R., Pflügers Arch. Physiol. 233, 486 (1934). - 9. Nachmansohn, D., in Chemical and molecular basis of nerve activity, Academic Press, New YorkLondon (1959). - 10. Engelhard, N., K. Prchal und M. Nenner, Angew. Chem. 79, 604 (1967). - 11. Green, A. L. und
H. J. Smith, Biochim. biophysica Acta Amsterdam 27, 212 (1958). 12. Hobbiger, F., Brit. J. Pharmacol. 12, 438 (1957). - 13. Wirson, I. B., Discuss. Faraday Soc. 20, 119 (1955). - 14. Davres, D. R. und A. L. GREEN, Biochem. J. 63, 529 (1956). - 15. HeILBRoNN, E., Biochem. Pharmacol. 12, 25 (1963). - 16. Flersher, J. H., L. W. Harris und E. F. Murtha, J. Pharmacol. Exper. Therap. Baltimoore 156, 345 (1967). - 17. BeRRY, W. K. und D. R. Davies, Biochem. J. 100, 572 (1966). - 18. Scarfe, J. F., Canad. J. Biochem. 37, 1301 (1959). - 19. ZeCH, R., H. ENGELHARD und W.-D. ERDMaNN, Biochem. biophysica Acta Amsterdam 128, 363 (1966). - 20. LAMB, J. C und G. M. Steinberg, Biochim. biophysica Acta Amsterdam 89, 171 (1964). - 21. LAMB, J. C., G. M. Steinberg und B. E. HACKLEY jr., Biochim. biophysica Acta Amsterdam 89, 174 (1964).

Dr. Peter Back 78 Freiburg im Breisgau Hugstetter Str. 55 